\title{
Dehydroepiandrosterone sulfate and dehydroepiandrosterone sulfate/cortisol ratio in cirrhotic patients with septic shock: another sign of hepatoadrenal syndrome?
}

Ming-Hung Tsai ${ }^{1 \dagger}$, Hui-Chun Huang ${ }^{2,3,4 \dagger}$, Yun-Shing Peng ${ }^{5,6}$, Yung-Chang Chen ${ }^{6,7}$, Ya-Chung Tian ${ }^{6,7}$, Chih-Wei Yang ${ }^{6,7}$, Jau-Min Lien ${ }^{1}$, Ji-Tseng Fang ${ }^{6,7}$, Cheng-Shyong Wu ${ }^{6,8}$, Sen-Yung Hsieh ${ }^{1}$ and Fa-Yauh Lee ${ }^{2,3^{*}}$

\begin{abstract}
Background: Cirrhotic patients are susceptible to sepsis and critical illness-related corticosteroid insufficiency (CIRCI). Dehydroepiandrosterone sulfate (DHEAS) is a corticotropin-dependent adrenal androgen, which has immunostimulating and antiglucocorticoid effects. Considering the synchronized synthesis of cortisol and DHEAS and their opposing effects to each other, investigators have proposed measuring these two hormones as a ratio. Severe sepsis has been associated with low DHEAS, especially relative to high cortisol. Despite growing interest in the role of adrenal androgen replacement in critical illness, there have been no data about DHEAS and the DHEAS/cortisol ratio in patients with liver cirrhosis. We studied whether low concentrations of DHEAS and decreased DHEAS/cortisol ratio are associated with poor outcome in patients with liver cirrhosis and septic shock.
\end{abstract}

Methods: We recruited 46 cirrhotic patients with septic shock, and 46 noncirrhotic counterparts matched by age and sex. We evaluated adrenal function using the short corticotropin stimulation test and analyzed the relation between DHEAS and cortisol.

Results: While the nonsurvivors in the cirrhotic group had significantly lower baseline DHEAS, lower baseline DHEAS/cortisol ratio, and reduced increments of both DHEAS and cortisol upon corticotropin stimulation, the survivors had lower baseline cortisol. Cirrhotic patients with lower DHEAS/cortisol ratio $(<1.50)$ had higher levels of interleukin-6 and tumor necrosis factor alpha, higher Sequential Organ Failure Assessment scores, and higher rates of $\mathrm{CIRCl}$ and hospital mortality. Using the area under the receiver operating characteristic (AUROC) curve, both DHEAS and the DHEAS/cortisol ratio demonstrated a good discriminative power for predicting hospital survival (AUROC 0.807 and 0.925 respectively). The cirrhotic group had lower DHEAS and DHEAS/cortisol ratio but higher rates of CIRCI and hospital mortality, compared to the noncirrhotic group.

Conclusions: There is dissociation between cortisol (increased) and DHEAS (decreased) in those cirrhotic patients who succumb to septic shock. Low DHEAS/cortisol ratios are associated with more severe diseases, inflammation, and CIRCI and can serve as a prognostic marker. More investigations are needed to evaluate the role of adrenal androgen in this clinical setting.

Keywords: Adrenal androgen, Steroidogenesis, Cirrhosis, Sepsis

\footnotetext{
* Correspondence: fylee@vghtpe.gov.tw

${ }^{\dagger}$ Equal contributors

${ }^{2}$ Division of Gastroenterology and Hepatology, Department of Internal

Medicine, Taipei Veteran General Hospital, No. 201, Section 2, Shih-Pai Road,

Taipei 11217, Taiwan

${ }^{3}$ Faculty of Medicine, Yang-Ming University School of Medicine, Taipei, Taiwan

Full list of author information is available at the end of the article
} 


\section{Background}

Septic shock is accompanied by activation of the hypothalamic-pituitary-adrenal (HPA) axis, which is highlighted by increased serum corticotropin and glucocorticoid [1-3]. Parallel to glucocorticoid secretion, HPA activation leads to the release of dehydroepiandrosterone (DHEA) and its sulfate (DHEAS). Both of these are adrenal androgens and practically all is secreted by the adrenal glands [4]. The serum concentration of DHEAS is 300-500 times higher than that of DHEA and can be considered a circulating reservoir [4]. In contrast to serum cortisol or DHEA concentrations, serum DHEAS levels do not exhibit a diurnal variation, as a consequence of a longer half-life [4]. Therefore, serum DHEAS levels have been proposed to serve as a potential biomarker of adrenal function in different clinical settings $[5,6]$. In fact, investigators have combined adrenocorticotropic hormone (ACTH) stimulation tests or insulin tolerance tests with measurements of DHEAS to define normality of the HPA axis $[7,8]$. Importantly, similar to that of glucocorticoid, the secretion of adrenal androgen is almost exclusively under the trophic effect of ACTH [4]. Normally, adrenal androgen is secreted synchronously with glucocorticoid from the adrenal cortex [9]. Although the specific physiological function of DHEA is still unclear, it has been shown that DHEA modulates the function of the immune system. DHEA plays an important role in the interaction between the endocrine and immune systems [10, 11]. The immune-modulatory properties of DHEA include induction of T-cell activation and interleukin-2 production [12, 13], and enhancing cytotoxic functions of monocytes [14]. Because of these immune-enhancing effects, adrenal androgen has been considered a functional antagonist of glucocorticoids, which can be immunosuppressive [4]. In fact, a recent study indicated that DHEA and glucocorticoids regulate the same immune-modulating genes in opposite directions [15]. Considering lines of evidence for activities of adrenal androgen that offset those of glucocorticoid, investigators have proposed quantification of their levels as a ratio, to serve as an alternative index of adrenal activity in different clinical settings [16, 17]. Interestingly, the responses of glucocorticoid and androgen demonstrate a significant discrepancy during critical illness, with adrenal androgen levels decreasing and glucocorticoid levels increasing [18]. This phenomenon is more pronounced in most severely ill patients and nonsurvivors [18-20], suggesting that functional adaptation of adrenal steroidogenesis may exhaust the counterregulatory mechanisms between adrenal androgen and glucocorticoid, thus negatively impacting the prognosis of critical illness. Indeed, an increased cortisol to DHEAS ratio was shown in nonsurvivors $[19,20]$, indicating that an exhausted adrenal reserve can serve as a prognostic factor.
Liver cirrhosis is significantly associated with an increased risk of sepsis and sepsis-related mortality $[21,22]$. Indeed, upregulated inflammatory cytokines, which mediate inflammation and organ dysfunction in the setting of liver cirrhosis and sepsis [22, 23], are major components facilitating interaction between immune and neuroendocrine systems [24]. Critical illness-related corticosteroid insufficiency (CIRCI) or relative adrenal insufficiency is common in cirrhotic patients with severe sepsis and septic shock, and is associated with increased mortality [25-28]. However, relevant data about adrenal androgen in critically ill cirrhotic patients are still lacking despite growing interest in the role of adrenal androgen replacement in critical illness [29]. In fact, decreased levels of adrenal androgen are reported in noncritical patients with liver cirrhosis [30, 31]. In a sense, overshooting inflammation in septic shock may overwhelm the adaptive strategy of adrenal steroidogenesis in those patients who succumb. We hypothesized that defective DHEAS production and adrenal exhaustion are associated with inflammation and poor prognosis in cirrhotic patients with septic shock. In this prospective observational investigation, we used the short corticotropin stimulation test (SST) to evaluate adrenal function and studied whether low concentrations of DHEAS and a decreased DHEAS/ cortisol ratio are associated with poor outcome in patients with liver cirrhosis and septic shock.

\section{Methods}

\section{Patient information, data collection, and definitions}

This study was conducted with the approval of the institutional review board of Chang Gung Memorial Hospital, Taiwan and in accordance with the Declaration of Helsinki of the World Medical Association. Written informed consent was obtained from the patients or from their legally accepted representatives for those with hepatic encephalopathy. From August 2010 to January 2012, 46 cirrhotic patients were consecutively enrolled and fulfilled the criteria of septic shock proposed by the members of American College of Chest Physicians/Society of Critical Care Medicine consensus conference committee [32], namely sepsis-induced hypotension despite adequate fluid resuscitation, along with the presence of hypoperfusion abnormalities associated with organ dysfunction. Sepsis-induced hypotension was defined as systolic blood pressure $<90 \mathrm{mmHg}$ or a reduction $<40 \mathrm{mmHg}$ from baseline in the absence of other causes for hypotension. Liver cirrhosis was defined histologically or based on clinical, image, and laboratory findings. The diagnosis was made histologically in eight patients in whom liver biopsy was performed to diagnose hepatocellular carcinoma or confirm liver cirrhosis. Liver cirrhosis was diagnosed clinically in 38 patients. A control group of 46 patients with septic shock without cirrhosis, matched 
by age and sex, was identified from patients admitted during the same period. All patients were resuscitated with a standard treatment protocol for management of septic shock [33].

The severity of liver disease on the day of the SST was graded by the Child-Pugh system and the Model for Endstage Liver Disease (MELD) [34, 35]. Meanwhile, illness severity was also assessed by Sequential Organ Failure Assessment (SOFA) [36]. For these scoring systems and physiological evaluations, the most abnormal value for each organ system on the day of the SST was recorded.

Patients with a history of corticosteroid treatment and those who had received the steroidogenesis-inhibiting agent etomidate over the preceding 6 months were excluded from this study. The main outcome analyzed was hospital mortality.

\section{Laboratory investigations}

Hematological and biochemical data were collected systemically on the day of the SST. Blood cultures, urine sediment, urine culture, ascitic fluid neutrophil count, and culture were performed routinely at inclusion. Blood cultures and appropriate culture specimens from the infection focus were obtained during hospitalization if necessary.

A SST was performed within the first 24 hours of admission to the ICU. Synthetic ACTH $(250 \mu$ g, Synacthen; Novartis Pharma AG, Basel, Switzerland) was given intravenously. Blood samples were obtained immediately before and 30 and 60 minutes after injection. Cortisol levels were measured by a competitive immunoassay using direct chemiluminescent technology (Roche Diagnostics, Mannhein, Germany). DHEAS levels were measured using a highly specific immunoradiometric assay (Nichols Institute Diagnostics, San Clemente, CA, USA). For cortisol measurement, the intra-assay coefficient of variation $(\mathrm{CV})$ was $2.8 \%$ and the inter-assay CV was $3.6 \%$. For DHEAS, the intra-assay CV was $8.6 \%$ and the inter-assay CV was $9.8 \%$. The peak levels of cortisol or DHEAS were defined as the highest levels obtained following synacthen administration, whether at 30 or 60 minutes. The cortisol or DHEAS increment was defined as the difference between the baseline and peak cortisol or DHEAS level. According to the consensus statements from an international task force [37], the criteria for CIRCI are defined as follows: baseline value $<10 \mu \mathrm{g} / \mathrm{dl}$ or cortisol response $<9 \mu \mathrm{g} / \mathrm{dl}$. The concentrations of TNF- $\alpha$ and IL- 6 were measured by an enzyme-linked immunosorbent assay ( $R$ \& D Systems, Minneapolis, MN, USA).

\section{Statistical analysis}

Descriptive statistics are expressed as mean \pm SD or median (interquartile range (IQR)). All variables were tested for normal distribution using the Kolmogorov-Smirnov test. The Student $t$ test was used to compare the means of continuous variables and the normal distribution data. Otherwise, the Mann-Whitney $U$ test was used. Categorical data were tested using the chi-square $\left(\chi^{2}\right)$ test. The correlation between the results of the SST and the disease severity scores was analyzed with linear regression using the Pearson method. Discrimination was tested using the area under a receiver operating characteristic (ROC) curve to assess the ability of DHEAS and the DHEAS/cortisol ratio to predict hospital survival. ROC analysis was also performed to calculate the cutoff values, sensitivity, specificity, overall correctness, and positive and negative predictive values. The best Youden index (sensitivity + specificity - 1) was also used to determine the best cutoff point of DHEAS and DHEAS/cortisol ratio to predict hospital survival. All statistical tests were two-tailed, and the significance level was set at $p=0.05$ or lower. Data were analyzed using SPSS 13.0 for Windows (SPSS Inc., Chicago, IL, USA) except for comparisons of ROC curves. The comparisons between ROC curves were calculated with MedCalc software (MedCalc Software 14.12.0, Belgium), using Hanley and McNeil's method [38].

\section{Results \\ Subjects' characteristics and short corticotropin stimulation test}

During the study period, 46 cirrhotic patients with septic shock were consecutively recruited and evaluated. Overall, the hospital mortality for the entire group was $73.9 \%$.

Table 1 presents patient demographic data, clinical characteristics, and results of the SST for both survivors and nonsurvivors. As shown in Table 1 and Fig. 1, both cortisol and DHEAS increments upon challenge of ACTH were significantly higher in those who survived. While the baseline cortisol levels were higher in those who died, the baseline DHEAS levels were significantly higher in those who survived. As a consequence, the baseline DHEAS/cortisol ratio was significantly lower in those who died. The peak cortisol levels were not different between survivors and nonsurvivors, while peak DHEAS levels were significantly lower in nonsurvivors. Both IL- 6 and TNF- $\alpha$ levels were significantly higher in nonsurvivors. The discriminating power of baseline DHEAS and DHEAS/cortisol ratio to predict hospital survival was tested using the area under a ROC curve. The areas under ROC curves (mean \pm SEM) for baseline DHEAS and DHEAS/cortisol ratio were $0.807 \pm 0.071$ (95\% CI: $0.668-0.947)$ and $0.925 \pm 0.039$ (95\% CI: 0.848-1.002) respectively. Comparison of AUROCs using Hanley and McNeil's method showed that the baseline DHEAS/cortisol ratio gave a significantly higher AUROC $(p=0.032)$ and thus better predictive accuracy than baseline DHEAS. Table 2 presents the predictive values of the chosen cutoff points $(755 \mathrm{nmol} / \mathrm{L}$ for DHEAS, 1.50 for 
Table 1 Patients' demographic data and clinical characteristics at admission to the ICU grouped according to hospital mortality

\begin{tabular}{llll}
\hline & Hospital survivors $(n=12)$ & Hospital nonsurvivors $(n=34)$ & $p$ value \\
\hline Age (years) & $47.6 \pm 14.8$ & $58.8 \pm 14.3$ & 0.029 \\
Gender (M/F) & $11 / 1$ & $25 / 9$ & 0.252 \\
SOFA score & $8.3 \pm 2.5$ & $14.0 \pm 3.6$ & $33.0 \pm 9.9$ \\
MELD score & $20.9 \pm 10.7$ & $12(11-13.3)$ & 0.001 \\
Child-Pugh score & $10(7.3-11.8)$ & $20.7(13.1-35.1)$ & 0.001 \\
ACTH (pg/ml) & $8.7(7.5-25.5)$ & $821(508-1312)$ & 0.005 \\
Baseline cortisol (nmol/L) & $484(381-796)$ & $993(807-1549)$ & 0.084 \\
Peak cortisol (nmol/L) & $955(633-1258)$ & $200(110-252)$ & 0.026 \\
Cortisol increment (nmol/L) & $359(257-428)$ & $622(403-1006)$ & 0.354 \\
Baseline DHEAS (nmol/L) & $1099(787-2627)$ & $699(429-1115)$ & 0.011 \\
Peak DHEAS (nmol/L) & $1286(1118-2874)$ & $41(10-96)$ & 0.002 \\
DHEAS increment (nmol/L) & $236(179-391)$ & $0.92 \pm 0.73$ & $<0.001$ \\
Baseline DHEAS/cortisol ratio & $2.86 \pm 1.40$ & $571(247.25-924)$ & 0.001 \\
IL-6 (pg/ml) & $110(54-233)$ & $45.5(31.25-78.25)$ & 0.001 \\
TNF-a (pg/ml) & $10(5.5-25)$ & & 0.001 \\
\hline
\end{tabular}

Data presented as mean $\pm S D$, number, or median (interquartile range)

$M$ male, $F$ female, SOFA Sequential Organ Failure Assessment, MELD Model for End-Stage Liver Disease, ACTH adrenocorticotropic hormone, DHEAS dehydroepiandrosterone sulfate, IL-6 interleukin-6, TNF-a tumor necrosis factor alpha, ICU intensive care unit

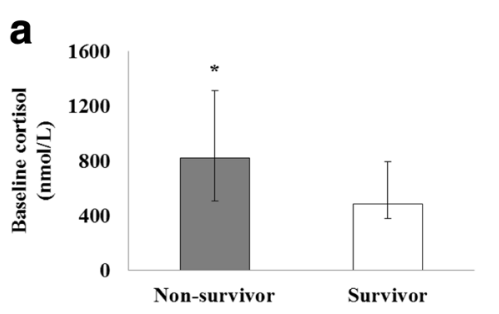

b

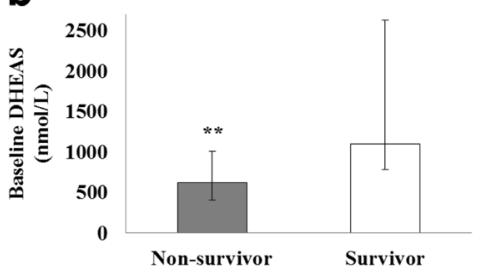

C

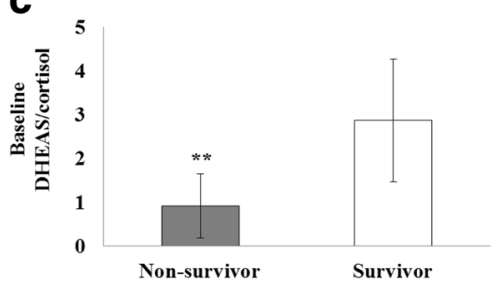

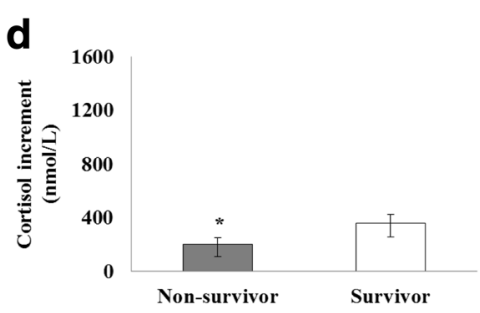

e

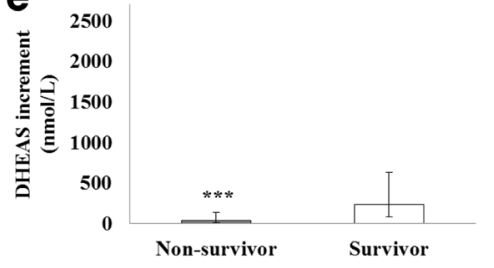

Fig. 1 Results of SSTs. a Levels of baseline cortisol are significantly higher in nonsurvivors. $\mathbf{b}$ Levels of baseline DHEAS are significantly higher in survivors. c Baseline DHEAS/cortisol ratios are significantly higher in survivors. $\mathbf{d}$ Cortisol increments upon challenge of ACTH are significantly higher in survivors. e DHEAS increments upon challenge of ACTH are significantly higher in survivors. Results expressed as median, error bars representing the interquartile range, in $\mathbf{a}, \mathbf{b}, \mathbf{d}$, e. Results expressed as mean, error bars representing the standard deviation, in $\mathbf{c} .{ }^{*} p<0.05,{ }^{* *} p<0.01,{ }^{* * *} p<0.001$. DHEAS dehydroepiandrosterone sulfate 
Table 2 Baseline DHEAS and DHEAS/cortisol ratio to predict hospital survival

\begin{tabular}{lccccc}
\hline & Sensitivity & Specificity & PPV & NPV & Accuracy \\
\hline DHEAS & 0.818 & 0.647 & 0.429 & 0.917 & 0.689 \\
DHEAS/cortisol & 0.909 & 0.842 & 0.625 & 0.966 & 0.844 \\
\hline
\end{tabular}

DHEAS dehydroepiandrosterone sulfate, PPV positive predictive value, NPV negative predictive value

DHEAS/cortisol ratio), which give the best Youden index, for prediction of hospital survival. The clinical characteristics and outcomes in patient subgroups stratified by DHEAS/cortisol ratio are presented in Table 3. As this table shows, a low DHEAS/cortisol ratio is associated with higher SOFA scores, inflammatory cytokine levels, rates of CIRCI, and hospital mortality.

According to the criteria stated, $28(60.8 \%)$ patients had CIRCI. The clinical characteristics and outcomes in patient subgroups stratified by adrenal functions are presented in Table 4. Those patients with CIRCI had lower baseline DHEAS levels and DHEAS/cortisol ratios and higher levels of inflammatory cytokines.

Cortisol and DHEAS increments were positively correlated $(R=0.378, p=0.010)$. Both cortisol and DHEAS increments were inversely correlated to SOFA score $(R=-0.517, p<0.001$ and $R=-0.476, p=0.001$ respectively), MELD score $(R=-0.444, p=0.002$ and $R=-0.382, \quad p=0.009$ respectively), and Child-Pugh score $(R=-0.546, p<0.001$ and $R=-0.368, p=0.012$ respectively). While baseline cortisol levels were inversely correlated to cortisol increments $(R=-0.382, p=0.009)$, baseline DHEAS levels were positively correlated to DHEAS increments $(R=0.521, p<0.001)$. The baseline DHEAS/cortisol ratio was negatively correlated to SOFA score $(R=-0.440, p=0.002)$ (Fig. 2a), MELD score $(R=-0.371, p=0.011)$, Child-Pugh score $(R=-0.471$, $p=0.001)$, IL-6 $(R=-0.503, p=0.005) \quad$ (Fig. $2 \mathrm{~b})$, and TNF- $\alpha(R=-0.634, p<0.001)$.

\section{Comparison between patients with septic shock with and without liver cirrhosis}

Table 5 presents the comparison between two groups of patients with and without cirrhosis matched by age and sex. Patients with liver cirrhosis and septic shock had significantly lower levels of baseline DHEAS and DHEAS/ cortisol ratios and significantly higher rates of hospital mortality. While the cirrhotic group had significantly lower increments of cortisol and DHEAS, the noncirrhotic group had lower levels of TNF- $\alpha$ and IL- 6 .

\section{Comparison between male and female patients with liver cirrhosis}

To address the gender effects on DHEAS biosynthesis, we stratified cirrhotic patients into male and female groups. As shown in Table 6, there was no difference in levels of baseline DHEAS, peak DHEAS, DHEAS increment, and baseline DHEAS/cortisol between male and female groups.

Table 3 Patients' demographic data and clinical characteristics grouped according to baseline DHEAS/cortisol ratio

\begin{tabular}{llll}
\hline & High DHEAS/cortisol ratio $(>1.50)(n=17)$ & Low DHEAS/cortisol ratio $(<1.50)(n=29)$ & $p$ value \\
\hline Age (years) & $48.7 \pm 11.4$ & $60.2 \pm 15.2$ & 0.010 \\
Gender (M/F) & $15 / 2$ & $21 / 8$ & 0.282 \\
Hospital mortality & $6 / 17(35.3 \%)$ & $28 / 29(96.6 \%)$ & $<0.001$ \\
SOFA score & $10.8 \pm 4.5$ & $13.6 \pm 3.7$ & $32.1 \pm 10.0$ \\
MELD score & $26.1 \pm 12.7$ & $12(11-13.5)$ & 0.030 \\
Child-Pugh score & $12(8-12.5)$ & $20.4(10.8-38)$ & 0.082 \\
ACTH (pg/ml) & $19(7.8-27.2)$ & $910(661-1422)$ & 0.106 \\
Baseline cortisol (nmol/L) & $441(382-691)$ & $1048(902-1671)$ & 0.372 \\
Peak cortisol (nmol/L) & $822(608-1141)$ & $196(97-288)$ & 0.001 \\
Cortisol increment (nmol/L) & $273(189-403)$ & $551(362-769)$ & 0.006 \\
Baseline DHEAS (nmol/L) & $1099(885-2124)$ & $643(360-1057)$ & 0.037 \\
Peak DHEAS (nmol/L) & $1286(974-2546)$ & $35.3(6.8-118.1)$ & $<0.001$ \\
DHEAS increment (nmol/L) & $135.7(88.2-248.3)$ & $571(221-1012)$ & $<0.001$ \\
IL-6 (pg/ml) & $198(58.5-444)$ & $47.5(30-96.8)$ & 0.009 \\
TNF-a (pg/ml) & $17.6(8.4-32.3)$ & $21 / 29(72.4 \%)$ & 0.025 \\
CIRCl & $7 / 17(41.2 \%)$ & & 0.002 \\
\hline
\end{tabular}

Data presented as mean $\pm \mathrm{SD}$, number (percentage), or median (interquartile range)

$M$ male, $F$ female, SOFA Sequential Organ Failure Assessment, MELD Model for End-Stage Liver Disease, ACTH adrenocorticotropic hormone,

DHEAS dehydroepiandrosterone sulfate, IL-6 interleukin-6, TNF-a tumor necrosis factor alpha, CIRCI critical illness-related corticosteroid deficiency 
Table 4 Patients' demographic data, clinical characteristics at admission to ICU and outcomes grouped according to the SST

\begin{tabular}{llll}
\hline & CIRCI $(n=28)$ & Non-CIRCI $(n=18)$ & $p$ value \\
\hline Age (years) & $56.0 \pm 12.8$ & $56.0 \pm 18.0$ & 0.997 \\
Gender (M/F) & $22 / 6$ & $14 / 4$ & 1.000 \\
SOFA score & $14.8 \pm 3.3$ & $8.7 \pm 2.8$ & $<0.001$ \\
MELD score & $35.3 \pm 8.8$ & $21.3 \pm 9.5$ & $<0.001$ \\
Child-Pugh score & $13(12-14)$ & $9(8-11.5)$ & $<0.001$ \\
ACTH (pg/ml) & $28(15-40)$ & $7.9(6.7-18.3)$ & $<0.001$ \\
Baseline cortisol (nmol/L) & $877(481-1331)$ & $55(417-859)$ & 0.054 \\
Peak cortisol (nmol/L) & $967(695-1505)$ & $1021(803-1458)$ & 0.727 \\
Cortisol increment (nmol/L) & $145(70-217)$ & $399(314-546)$ & $<0.001$ \\
Baseline DHEAS (nmol/L) & $678(437-1111)$ & $921(524-1552)$ & 0.229 \\
Peak DHEAS (nmol/L) & $750(491-1141)$ & $1159(595-1866)$ & 0.075 \\
DHEAS increment (nmol/L) & $41(16-101)$ & $180(90-278)$ & 0.002 \\
Baseline DHEAS/cortisol & $1.01 \pm 0.79$ & $2.11 \pm 1.58$ & 0.012 \\
IL-6 (pg/ml) & $660(240-968)$ & $164(85-328)$ & 0.011 \\
TNF-a (pg/ml) & $44(31-79)$ & $16(6-36)$ & 0.007 \\
Hospital mortality & $26 / 28(92.9 \%)$ & $8 / 18(44.4 \%)$ & $<0.001$ \\
\hline Data & &
\end{tabular}

Data presented as mean $\pm S D$, number (percentage), or median (interquartile range)

$M$ male, $F$ female, SST short corticotropin stimulation test, CIRCI critical illness-related corticosteroid deficiency, SOFA Sequential Organ Failure Assessment, MELD Model for End-Stage Liver Disease, ACTH adrenocorticotropic hormone, DHEAS dehydroepiandrosterone sulfate, IL-6 interleukin-6, TNF-a tumor necrosis factor alpha, ICU intensive care unit

\section{Discussion}

The major findings of this study are as follows. First, the ratio of DHEAS (an immunostimulant) to cortisol (an immunosuppressant) is significantly lower in patients with liver cirrhosis and septic shock, compared to their noncirrhotic counterparts. Second, upon admission to the ICU there is a dissociation between baseline DHEAS (reduced) and cortisol (increased) in nonsurvivors of the cirrhotic group, suggesting a functional adaptation of adrenal steroidogenesis in this subgroup. Finally, upon admission to the ICU a low DHEAS/cortisol ratio is associated with CIRCI and hospital mortality. The DHEAS/cortisol ratio can serve as a prognostic marker in this clinical setting.

Considering the synchronized synthesis of cortisol and DHEAS and their opposing effects to each other, there has been growing interest in measuring these two hormones as a ratio rather than as separate values $[16,17]$. In this regard, our study is the first to measure the ratio of DHEAS to cortisol in liver cirrhosis. Interestingly, we found that a low DHEAS/cortisol ratio is associated with inflammation, disease severity, CIRCI, and hospital mortality (Table 3). Our results suggest that the balance between these two mutually dependent hormones may influence the pathophysiological process and ultimately outcomes. Indeed, this index has been used as an indicator in different diseases [16, 17, 19, 20]. This approach also helps to conceptualize the preferential synthesis of glucocorticoid over adrenal androgen in liver cirrhosis and septic shock. Previous studies have shown that there is a discrepancy between DHEAS (decreased) and cortisol (increased) in critical illness [18]. In accordance with this notion, we showed that nonsurvivors of the cirrhotic group had significantly lower baseline DHEAS and significantly higher baseline cortisol, and thus a lower DHEAS/cortisol ratio. The combination of low DHEAS levels with high cortisol levels suggested a shift in pregnenolone metabolism away from adrenal androgen pathways toward the glucocorticoid pathway in this subset of patients. It is conceivable that this functional adaptation could serve to minimize the synthesis of adrenal androgen and maximize the secretion of glucocorticoid which is acutely necessary to antagonize inflammation. Other intriguing findings are that baseline cortisol levels were negatively correlated to cortisol increments, whereas baseline DHEAS levels were positively correlated to DHEAS increments. Furthermore, both DHEAS and cortisol increment were negatively correlated to disease severity scores and associated with hospital mortality. Taken together, preferential adaptation of adrenal steroidogenesis favoring glucocorticoid pathway may deplete adrenal reserve and exhaust the counterbalance between adrenal androgen and glucocorticoid, thus negatively impacting the prognosis of liver cirrhosis with septic shock. 

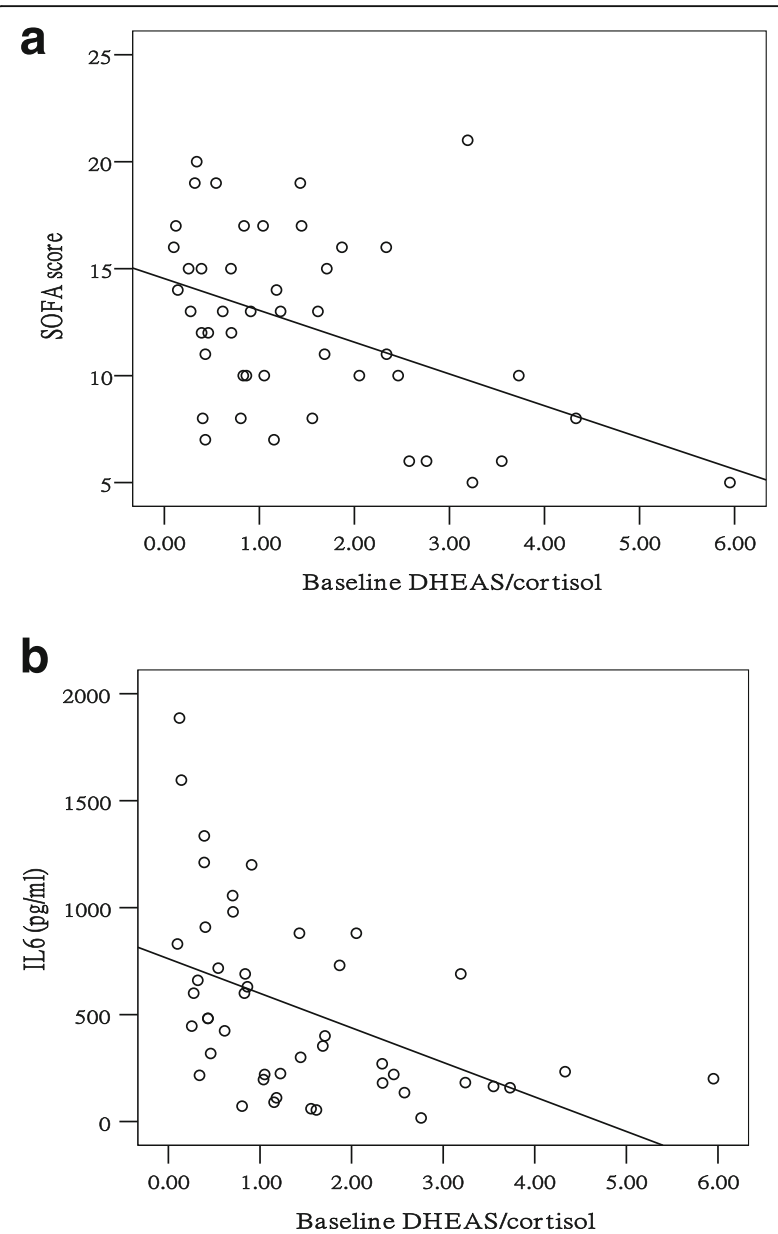

Fig. 2 a Baseline DHEAS/cortisol ratio is negatively correlated to SOFA score $(R=-0.440, p=0.002)$. b Baseline DHEAS/cortisol ratio is negatively correlated to IL-6 $(R=-0.503, p=0.005)$. SOFA Sequential Organ Failure Assessment, DHEAS dehydroepiandrosterone sulfate, IL6 interleukin-6

Adrenal androgens are pleiotropic hormones with multiple biological functions. In addition to the immune-enhancing effects mentioned previously, adrenal androgen has been shown to modulate cardiovascular functions. DHEA can exert its protective effects on the cardiac function and integrity of microcirculation of different vasculatures through activating Akt-eNOS signaling pathways [39-42]. Indeed, in experimental animals subjected to sepsis, administration of DHEA can attenuate organ dysfunction and improve survival. This effect was paralleled by decreased inflammatory cytokine levels and an improved activity of T-cell-mediated immunity [10, 43]. Moreover, salutary effects on hepatic perfusion were also demonstrated in experimental animals treated with androstenediol, a metabolite of DHEA, and subjected to trauma-hemorrhagic shock [44]. Interestingly, these beneficial effects of androstenediol on intrahepatic hemodynamics are due to induction of eNOS-mediated $\mathrm{NO}$ and a decrease in endothelin-1 [44], which may also represent a potential remedy for endothelial dysfunction in the microcirculation of cirrhotic liver [45]. It is unknown whether adrenal androgen can provide beneficial effects on immunity and microcirculation and thus improve outcomes in liver cirrhosis with septic shock.

Recently, CIRCI or relative adrenal insufficiency has been used to describe a suboptimal adrenal response to ACTH in critical illness, in which the glucocorticoid levels, although high in terms of absolute value, are inadequate to control inflammation [1, 37]. In most studies, only glucocorticoid levels were measured although the adrenal cortex secretes androgen as well as glucocorticoid upon ACTH stimulation [4]. In this study, we showed that CIRCI is associated with significantly lower DHEAS/cortisol ratio and significantly higher inflammatory cytokines and hospital mortality (Table 4). Additionally, the DHEAS/cortisol ratio had excellent capacity to predict hospital survival in cirrhotic patients with septic shock $(A U R O C=0.925)$, suggesting that these two tightly coordinated hormones should be assessed in concert to better reflect adrenal dysfunction. Although administration of low doses of glucocorticoid in septic patients can restore vascular hyporeactivity [46] and reverse the shock status [26, 47-50], its impact on survival remains controversial $[26,48-50]$. The reasons for the discrepancy are unclear. Indeed, there are more episodes of superinfection, and higher rates of severe hyperglycemia associated with glucocorticoid administration $[26,49,50]$. In this regard, DHEA has been shown to enhance immunity and help overcome the catabolic effects of glucocorticoid [29]. It is plausible to speculate that glucocorticoid treatment alone may be incomplete unless the imbalance of steroidogenesis is offset by adrenal androgen in addition to glucocorticoid. Further studies are needed to clarify this issue.

Finally, another finding deserving discussion is that cirrhotic patients with septic shock had significantly lower baseline DHEAS, lower baseline DHEAS/cortisol ratio, and decreased cortisol and DHEAS increments upon ACTH challenge, when compared to the noncirrhotic group. However, the baseline cortisol levels were comparable between both groups. This phenomenon can be interpreted as a shift of steroidogenesis toward glucocorticoid biosynthesis at the expense of adrenal androgen in liver cirrhosis. We speculate that liver cirrhosis per se represents a risk factor for altered adrenal steroidogenesis, which may portend a poor prognosis for septic shock if upregulation of glucocorticoid becomes unopposed by adrenal androgen but is still insufficient to control inflammation. In this regard, glucocorticoid 
Table 5 Comparison between cirrhotic and noncirrhotic groups matched according to age and sex

\begin{tabular}{llll}
\hline & Liver cirrhosis $(n=46)$ & Nonliver cirrhosis $(n=46)$ & $p$ value \\
\hline Age (years) & $56.0 \pm 14.9$ & $58.1 \pm 20.5$ & 0.556 \\
Gender (M/F) & $36 / 10$ & $36 / 10$ & 1.000 \\
SOFA score & $12.7 \pm 4.1$ & $6.7 \pm 4.1$ & $<0.001$ \\
Baseline cortisol (nmol/L) & $748(450-1203)$ & $778(590-1109)$ & 0.628 \\
Peak cortisol (nmol/L) & $993(744-1479)$ & $1195(948-1589)$ & 0.041 \\
Cortisol increment (nmol/L) & $221(121-348)$ & $408(285-570)$ & $<0.001$ \\
ACTH (pg/ml) & $19.1(9.1-34.0)$ & $20.3(8-30.5)$ & 0.842 \\
Baseline DHEAS (nmol/L) & $703(464-1180)$ & $842(587-1432)$ & $<0.001$ \\
Peak DHEAS (nmol/L) & $939(497-1296)$ & $1037(643-1752)$ & $<0.001$ \\
DHEAS increment (nmol/L) & $89.6(23-185)$ & $325.7(174-546)$ & $<0.001$ \\
Baseline DHEAS/cortisol & $1.40 \pm 1.24$ & $3.86 \pm 2.57$ & 0.001 \\
IL-6 (pg/ml) & $424(206-855)$ & $88(35-275)$ & 0.002 \\
TNF-a (pg/ml) & $34(21-70)$ & $15(10.65-23.5)$ & 0.003 \\
Hospital mortality & $34 / 46(73.9 \%)$ & $16 / 46(34.8 \%)$ & $<0.001$ \\
\hline
\end{tabular}

Data presented as mean $\pm S D$, number (percentage), or median (interquartile range)

$M$ male, $F$ female, SOFA Sequential Organ Failure Assessment, ACTH adrenocorticotropic hormone, DHEAS dehydroepiandrosterone sulfate, IL-6 interleukin-6,

TNF-a tumor necrosis factor alpha

resistance may contribute to adrenal dysfunction in our patients. Finally, hepatoadrenal syndrome [27, 28, 51] may need to be redefined and addressed in the context of coordination between separate adrenal hormones.

There are limitations in our study. First, the number of patients is small. Second, the association does not indicate causality because of the observational design. Further studies with a larger cohort and therapeutic intervention are needed in the future.

\section{Conclusions}

We demonstrated a divergent biosynthesis between cortisol and DHEAS in cirrhotic patients with septic

Table 6 Patients' demographic data and clinical characteristics at admission to ICU grouped according to gender

\begin{tabular}{llll}
\hline & Male $(n=36)$ & Female $(n=10)$ & $p$ value \\
\hline Age (years) & $52.9 \pm 12.6$ & $67.0 \pm 17.9$ & 0.007 \\
Hospital mortality & $25 / 36(69.4 \%)$ & $9 / 10(90 \%)$ & $(0.252)$ \\
SOFA score & $12.2 \pm 4.4$ & $13.0 \pm 4.2$ & $(0.618)$ \\
MELD score & $30.5(24-39.8)$ & $27(16.8-37.8)$ & $(0.431)$ \\
Child-Pugh score & $12(10.3-13)$ & $11.5(9-13.3)$ & $(0.666)$ \\
ACTH (pg/ml) & $19.1(9.8-32.7)$ & $22(6.3-47.9)$ & $(0.929)$ \\
Baseline cortisol (nmol/L) & $675(428-1086)$ & $975(680-1260)$ & $(0.132)$ \\
Peak cortisol (nmol/L) & $927(697-1381)$ & $1338(955-1639)$ & $(0.088)$ \\
Cortisol increment (nmol/L) & $221(112-353)$ & $240(167-466)$ & $(0.432)$ \\
Baseline DHEAS (nmol/L) & $704(437-1140)$ & $824(510-1428)$ & $(0.409)$ \\
Peak DHEAS (nmol/L) & $877(491-1263)$ & $961(510-1692)$ & $(0.489)$ \\
DHEAS increment (nmol/L) & $89.6(25.8-171.7)$ & $70.6(0-245.7)$ & $(0.957)$ \\
Baseline DHEAS/cortisol ratio & $1.46 \pm 1.16$ & $1.37 \pm 1.68$ & $(0.843)$ \\
IL-6 (pg/ml) & $318(150-855)$ & $482(310-1288)$ & $(0.404)$ \\
TNF-a (pg/ml) & $31(11.2-78)$ & $47(39-52.5)$ & $(0.208)$ \\
CIRCl & $22 / 36(61.1 \%)$ & $6 / 10(60 \%)$ & $(1.000)$ \\
\hline Dia pres) &
\end{tabular}

Data presented as mean $\pm S D$, number (percentage), or median (interquartile range)

SOFA Sequential Organ Failure Assessment, MELD Model for End-Stage Liver Disease, ACTH adrenocorticotropic hormone, DHEAS dehydroepiandrosterone sulfate, IL-6 interleukin-6, TNF-a tumor necrosis factor alpha, CIRCI critical illness-related corticosteroid deficiency, ICU intensive care unit 
shock. This phenomenon, as evidenced by an altered DHEAS/cortisol ratio, is associated with more severe diseases and higher levels of inflammatory cytokines and can serve as a prognostic marker. More investigations are needed to evaluate the role of adrenal androgen in this clinical setting.

\section{Abbreviations}

CIRCI: Critical illness-related corticosteroid insufficiency;

DHEAS: Dehydroepiandrosterone sulfate; HPA: Hypothalamic-pituitary-adrenal; ICU: Intensive care unit; MELD: Model for End-Stage Liver Disease; SOFA: Sequential Organ Failure Assessment; SST: Short corticotropin stimulation test

\section{Acknowledgements}

The authors gratefully acknowledge the technical assistance of I-Shyann Huang.

\section{Funding}

This work is partially supported by grants from Chang Gung Medical Research Fund CMRPG3D0191, and Ministry of Science and Technology Research fund 102-2314B-182A-025-, Taiwan.

\section{Availability of data and materials}

The datasets used and/or analyzed during the current study are available from the corresponding author on reasonable request.

\section{Authors' contributions}

F-YL conceived the study and approved the final version of the manuscript $\mathrm{M}-\mathrm{HT}$ and $\mathrm{H}-\mathrm{CH}$ participated in study conception, design, and coordination and drafted the manuscript. Y-SP, Y-CC, Y-CT, C-WY, J-ML, J-TF, C-SW, and $\mathrm{S}-\mathrm{YH}$ participated in collection, analysis, and interpretation of data. All authors read and approved the final manuscript.

\section{Ethics approval and consent to participate}

This study was conducted with the approval of the institutional review board of Chang Gung Memorial Hospital, Taiwan and in accordance with the Declaration of Helsinki of the World Medical Association. Written informed consent was obtained from the patients or from their legally accepted representatives in those with hepatic encephalopathy.

\section{Consent for publication}

Not applicable.

\section{Competing interests}

The authors declare that they have no competing interests.

\section{Publisher's Note}

Springer Nature remains neutral with regard to jurisdictional claims in published maps and institutional affiliations.

\section{Author details \\ 'Division of Gastroenterology and Hepatology, Chang Gung Memorial Hospital, Chang Gung University, Taoyuan, Taiwan. ${ }^{2}$ Division of Gastroenterology and Hepatology, Department of Internal Medicine, Taipei Veteran General Hospital, No. 201, Section 2, Shih-Pai Road, Taipei 11217, Taiwan. ${ }^{3}$ Faculty of Medicine, Yang-Ming University School of Medicine, Taipei, Taiwan. ${ }^{4}$ Division of General Medicine, Department of Medicine, Taipei Veteran General Hospital, Taipei, Taiwan. ${ }^{5}$ Division of Endocrinology, Chang Gung Memorial Hospital, Chia-Yi, Taiwan. ${ }^{6}$ Chang Gung University, Taoyuan, Taiwan. ${ }^{7}$ Division of Critical Care Nephrology, Kidney Institute, Chang Gung Memorial Hospital, Taipei, Taiwan. ${ }^{8}$ Division of Gastroenterology, Chang Gung Memorial Hospital, Chia-Yi, Taiwan.}

Received: 28 February 2017 Accepted: 20 June 2017

Published online: 15 August 2017

\section{References}

1. Cooper MS, Stewart PM. Corticosteroid insufficiency in acutely ill patients. N Engl J Med. 2003;348:727-34
2. Jurney TH, Cockrell Jr JL, Lindberg JS, et al. Spectrum of serum cortisol response to ACTH in ICU patients. Correlation with degree of illness and mortality. Chest. 1987;92:292-5.

3. Reincke M, Allolio B, Wurth $\mathrm{G}$, et al. The hypothalamic-pituitary-adrenal axis in critical illness: response to dexamethasone and corticotropin-releasing hormone. J Clin Endocrinol Metab. 1993:77:151-6.

4. Kroboth PD, Salek FS, Pittenger AL, et al. DHEA and DHEA-S: a review. J Clin Pharmacol. 1999:39:327-48.

5. Leowattana W. DHEAS as a new diagnostic tool. Clin Chim Acta. 2004;341:1-15.

6. Al-Aridi R, Abdelmannan D, Arafah BM. Biochemical diagnosis of adrenal insufficiency: the added value of dehydroepiandrosterone sulfate measurements. Endocr Pract. 2011;17:261-70.

7. Nasrallah MP, Arafah BM. The value of dehydroepiandrosterone sulfate measurements in the assessment of adrenal function. J Clin Endocrinol Metab. 2003:88:5293-8.

8. Fischli S, Jenni S, Allemann S, et al. Dehydroepiandrosterone sulfate in the assessment of the hypothalamic-pituitary-adrenal axis. J Clin Endocrinol Metab. 2008:93:539-42.

9. Rosenfeld RS, Hellman L, Gallagher TF. Metabolism and inter-conversion of dehydroisoandrosterone and dehydroisoandrosterone sulfate. J Clin Endocrinol Metab. 1972;35:187-93.

10. Oberbeck R, Dahlweid M, Koch R, et al. Dehydroepiandrosterone decreases mortality rate and improves cellular immune function during polymicrobial sepsis. Crit Care Med. 2001;29:380-4.

11. Angele MK, Catania RA, Ayala A, et al. Dehydroepiandrosterone: an inexpensive steroid hormone that decreases the mortality due to sepsis following trauma-induced hemorrhage. Arch Surg. 1998;133:1281-8.

12. Suzuki T, Suzuki N, Daynes RA, et al. Dehydroepiandrosterone enhances IL2 production and cytotoxic effector function of human T cells. Clin Immunol Immunopathol. 1991;61:202-11.

13. Padgett DA, Loria RM. In vitro potentiation of lymphocyte activation by dehydroepiandrosterone, androstenediol, and androstenetriol. J Immunol. 1994; 153:1544-52

14. McLachlan JA, Serkin CD, Bakouche O. Dehydroepiandrosterone modulation of lipopolysaccharide-stimulated monocyte cytotoxicity. J Immunol. 1996; 156:328-35.

15. Maurer M, Trajanoski Z, Frey G, et al. Differential gene expression profile of glucocorticoids, testosterone, and dehydroepiandrosterone in human cells. Hormone Metab Res. 2002;33:691-5.

16. Bozza V, D'Attilio L, Mahuad CV, et al. Altered cortisol/DHEA ratio in tuberculosis patients and its relationship with abnormalities in the mycobacterial-driven cytokine production by peripheral blood. Scand J Immunol. 2007;66:97-103.

17. Mocking R, Pellikaan C, Lok A, et al. DHEAS and cortisol/DHEAS-ratio in recurrent depression: state, or trait predicting 10-year recurrence? Psychoneuroendocrinology. 2015;59:91-101.

18. Beishuizen A, Thijs LG, Vermes I. Decreased levels of dehydroepiandrosterone sulphate in severe critical illness: a sign of exhausted adrenal reserve? Crit Care. 2002;6:434-8.

19. Arlt W, Hammer F, Sanning P, et al. Dissociation of serum dehydroepiandrosterone and dehydroepiandrosterone sulfate in septic shock. J Clin Endocrinol Metab. 2006;91:2548-54.

20. Marx C, Petros S, Bornstein SR, et al. Adrenocortical hormones in survivors and nonsurvivors of severe sepsis: diverse time course of dehydroepiandrosterone, dehydroepiandrosterone-sulfate, and cortisol. Crit Care Med. 2003;31:1382-8.

21. Foreman MG, Mannino DM, Moss M. Cirrhosis as a risk factor for sepsis and death: analysis of the National Hospital Discharge Survey. Chest. 2003;124:1016-20.

22. Tandon P, Garcia-Tsao G. Bacterial infections, sepsis, and multiorgan failure in cirrhosis. Semin Liver Dis. 2008;28:26-42.

23. Tsai MH, Peng YS, Chen YC, et al. Low serum concentration of apolipoproteinA-I is an indicator of poor prognosis in cirrhotic patients with severe sepsis. J Hepatol. 2009;50:906-15.

24. Haddad JJ, Saadé NE, Safieh-Garabedian B. Cytokines and neuro-immuneendocrine interactions: a role for the hypothalamic-pituitary-adrenal revolving axis. J Neuroimmunol. 2002;133:1-19.

25. Tsai MH, Peng YS, Chen YC, et al. Adrenal insufficiency in patients with cirrhosis, severe sepsis and septic shock. Hepatology. 2006:43:673-81.

26. Fernández J, Escorsell A, Zabalza M, et al. Adrenal insufficiency in patients with cirrhosis and septic shock: effect of treatment with hydrocortisone on survival. Hepatology. 2006:44:1288-95. 
27. O'Beirne J, Holmes M, Agarwal B, et al. Adrenal insufficiency in liver disease-what is the evidence? J Hepatol. 2007;47:418-23.

28. Fede G, Spadaro L, Tomaselli T, et al. Adrenocortical dysfunction in liver disease: a systematic review. Hepatology. 2012;55:1282-91.

29. Dhatariya KK. Is there a role for dehydroepiandrosterone replacement in the intensive care population? Intensive Care Med. 2003;29:1877-80.

30. Franz C, Watson D, Longcope C. Estrone sulfate and dehydroepiandrosterone sulfate concentrations in normal subjects and men with cirrhosis. Steroids. 1979;34:563-73.

31. Kalaitzakis E, Josefsson A, Castedal M, et al. Factors related to fatigue in patients with cirrhosis before and after liver transplantation. Clin Gastroenterol Hepatol. 2012;10:174-81.

32. Bone RC, Balk RA, Cerra FB, et al. Definitions for sepsis and organ failure and guidelines for the use of innovative therapies in sepsis [The ACCP/SCCM consensus conference committee. American College of Chest Physicians/ Society of Critical Care Medicine]. Chest. 1992;101:1644-55.

33. Dellinger RP, Carlet JM, Masur $\mathrm{H}$, et al. Surviving sepsis campaign quidelines for management of severe sepsis and septic shock. Crit Care Med. 2004;32:858-73.

34. Pugh RN, Murray-Lyon IM, Dawson JL, et al. Transection of the esophagus in the bleeding esophageal varices. Br J Surg. 1973;60:648-52.

35. Kamath PS, Weisner RH, Malinchoc $\mathrm{M}$, et al. A model to predict survival in patients with end-stage liver disease. Hepatology. 2001;33:464-70.

36. Wehler M, Kokoska J, Reulbach U, et al. Short-term prognosis in critically ill patients with cirrhosis assessed by prognostic scoring systems. Hepatology. 2001;34:255-61.

37. Marik PE, Pastores SM, Annane D, et al. American College of Critical Care Medicine: Recommendations for the diagnosis and management of corticosteroid insufficiency in critically ill adult patients: consensus statements from an international task force by the American College of Critical Care Medicine. Crit Care Med. 2008;36:1937-49.

38. Hanley JA, McNeil BJ. A method of comparing the areas under receiver operating characteristic curves derived from the same cases. Radiology. 1983;148:839-48

39. Liu D, Si H, Reynolds KA, et al. Dehydroepiandrosterone protects vascular endothelial cells against apoptosis through Gai protein-dependent activation of phosphatidylinositol 3-kinase/Akt and regulation of antiapoptotic BCl-2 expression. Endocrinology. 2007;148:3068-76.

40. Liu D, Dillon JS. Dehydroepiandrosterone activates endothelial cell nitricoxide synthase by a specific plasma membrane receptor coupled to Gai2,3. J Biol Chem. 2002;277:21379-88.

41. Bhuiyan S, Fukunaga K. Stimulation of Sigma-1 receptor by dehydroepiandrosterone ameliorates hypertension-induced kidney hypertrophy in ovariectomized rats. Exp Biol Med (Maywood). 2010;235:356-64

42. Tagashira H, Bhuiyan S, Shioda N, et al. Distinct cardioprotective effects of $17 \beta$-estradiol and dehydroepiandrosterone on pressure overload-induced hypertrophy in ovariectomized female rats. Menopause. 2011;18:1317-26.

43. Danenberg HD, Alpert G, Lustig S, et al. Dehydroepiandrosterone protects mice fromendotoxin toxicity and reduces tumor necrosis factor production. Antimicrob Agents Chemother. 1992;36:2275-9.

44. Shimizu T, Szalay L, Choudhry MA, et al. Mechanism of salutary effects of androstenediol on hepatic function after trauma-hemorrhage: role of endothelial and inducible nitric oxide synthase. Am J Physiol Gastrointest Liver Physiol. 2005;288:G244-50.

45. Bosch J, Groszmann RJ, Shah VH. Evolution in the understanding of the pathophysiological basis of portal hypertension: how changes in paradigm are leading to successful new treatments. J Hepatol. 2015;62(1 Suppl):S121-30.

46. Bellissant $E$, Annane D. Effect of hydrocortisone on phenylephrine-mean arterial pressure dose-response relationship in septic shock. Clin Pharmacol Ther. 2000;68:293-303.

47. Briegel J, Forst $\mathrm{H}$, Haller $\mathrm{M}$, et al. Stress doses of hydrocortisone reverse hyperdynamic septic shock: a prospective, randomized, double-blind, single-center study. Crit Care Med. 1999;27:723-32.

48. Annane D, Sébille V, Charpentier C, et al. Effect of treatment with low doses of hydrocortisone and fludrocortisone on mortality in patients with septic shock. JAMA. 2002;288:862-71.

49. Sprung CL, Annane D, Keh D, CORTICUS Study Group, et al. Hydrocortisone therapy for patients with septic shock. N Engl J Med. 2008:358:111-24.

50. Arabi YM, Aljumah A, Dabbagh O, et al. Low-dose hydrocortisone in patients with cirrhosis and septic shock: a randomized controlled trial. CMAJ. 2010;182:1971-7.

51. Marik PE, Gayowski T, Starzl TE, et al. The hepatoadrenal syndrome: a common yet unrecognized clinical condition. Crit Care Med. 2005;33:1254-9.

\section{Submit your next manuscript to BioMed Central and we will help you at every step:}

- We accept pre-submission inquiries

- Our selector tool helps you to find the most relevant journal

- We provide round the clock customer support

- Convenient online submission

- Thorough peer review

- Inclusion in PubMed and all major indexing services

- Maximum visibility for your research

Submit your manuscript at www.biomedcentral.com/submit 